\title{
Role of serum albumin and proteinuria in patients with SARS-CoV-2 pneumonia
}

\author{
Cinzia Bassoli $^{1}$ | Letizia Oreni $^{2}$ | Elisabetta Ballone ${ }^{3}$ | Antonella Foschi ${ }^{2}$ | \\ Andrea Perotti $^{3}$ | Annalisa Mainini ${ }^{2}$ | Giacomo Casalini ${ }^{1}$ | Laura Galimberti $^{2}$ | \\ Luca Meroni $^{2}$ | Spinello Antinori ${ }^{1,2}$ | Laura Milazzo ${ }^{2}$ (I)
}

${ }^{1}$ Luigi Sacco Department of Biomedical and Clinical Sciences, Università degli Studi di

Milano, Milan, Italy

${ }^{2}$ Department of Infectious Diseases, ASST

Fatebenefratelli Sacco, Milan, Italy

${ }^{3}$ Department of Anesthesiology and Intensive Care, ASST Fatebenefratelli Sacco, Luigi Sacco Hospital, Milan, Italy

\section{Correspondence}

Laura Milazzo, Department of Infectious Diseases, ASST Fatebenefratelli Sacco, Milan, Italy, Via GB Grassi 74, 20157, Milan, Italy.

Email: laura.milazzo@unimi.it

\begin{abstract}
Background: Hypoalbuminemia is frequently observed in patients with SARS-CoV-2 infection although its underlying mechanism and relationship with the clinical outcome still need to be clarified.

Methods: We retrospectively evaluated in patients with COVID-19 hospitalised at the Fatebenefratelli-Sacco Hospital in Milan, the prevalence of hypoalbuminemia, its association with the severity of COVID-19, with the levels of C-reactive protein, d-dimer and interleukin-6 and with clinical outcome over a follow-up period of 30 days. Urinalysis was evaluated in a subgroup of patients.

Results: Serum albumin levels $<30 \mathrm{~g} / \mathrm{L}$ were found in 105/207 (50.7\%) patients at hospital admission. Overall, the median albumin value was $29.5 \mathrm{~g} / \mathrm{L}$ (IQR 25-32.8). A negative association was found between albumin levels and severity of COVID-19 $(P<.0001)$ and death $(P=.003)$. An inverse correlation was observed between albumin and both C-reactive protein and D-dimer at hospital admission ( $r=-.487$ and $r=-.479$, respectively; $P<.0001)$. Finally, a positive correlation was found between albumin levels and eGFR $(r=.137 ; P=.049)$. Proteinuria was observed in $75 \%$ of patients with available data and it did not differ between patients with hypoalbuminemia and those with albumin $\geq 30 \mathrm{~g} / \mathrm{L}(81 \%$ and $67 \%$, respectively; $P=.09)$.

Conclusion: In patients with COVID-19, hypoalbuminemia is common and observed in quite an early stage of pulmonary disease. It is strictly associated with inflammation markers and clinical outcome. The common finding of proteinuria, even in the absence of creatinine increase, indicates protein loss as a possible biomarker of local and systemic inflammation worthwhile to evaluate disease severity in COVID-19.
\end{abstract}

\section{1 | INTRODUCTION}

Hypoalbuminemia $(<32 \mathrm{~g} / \mathrm{L})$ is amongst the most frequently observed laboratory abnormalities in patients with SARS-CoV-2 infection ${ }^{1-4}$ and it was more pronounced in severe than mild-moderate cases. ${ }^{1,2}$ The underlying mechanism of hypoalbuminemia and its relationship with respiratory failure and clinical outcome still needs to be clarified.
We retrospectively evaluated in our cohort of patients with COVID-19 hospitalised at the Infectious Disease Ward (IDW) or intensive care unit (ICU) of Fatebenefratelli-Sacco Hospital in Milan, the prevalence of hypoalbuminemia and its association with the severity of COVID-19, the levels of C-reactive protein, d-dimer and interleukin- 6 levels and the clinical outcome over a follow-up period of 30 days. 


\section{PATIENTS AND METHODS}

The following data were retrieved from clinical charts at presentation: demographic data, concomitant diseases, severity of clinical presentation, fraction of inspired oxygen (FiO2), peripheral oxygen saturations, serum albumin levels, serum creatinine, levels of D-dimer and C-reactive protein (CRP), serum interleukin-6 (IL-6). Estimated glomerular filtration rate (eGFR) was calculated with the Modification of Diet in Renal Disease Study (MDRD) equation. ${ }^{5}$ For those patients with available urinalysis, data on proteinuria, hematuria and urine leukocytes were collected. Serum albumin measurements were available for all the patients at three time-points within the first week of hospitalisation (admission, at day 3 and day 7 from admission) and were registered. The patients with a history of kidney diseases were excluded from this study.

According to the Chinese Guidelines for the Diagnosis and Treatment of Novel Coronavirus (2019-nCoV) Infection, the severity of SARS-CoV-2 disease was classified into four grades: (a) mild, with slight clinical symptoms and no evidence of pneumonia; (b) moderate, with fever, respiratory symptoms and confirmed pneumonia; (c) severe, with any of the following: respiratory distress with $\mathrm{RR}>30$ times/minutes, oxygen saturation at rest $<93 \%$ or $\mathrm{PaO} 2 / \mathrm{FiO} 2<300 \mathrm{mmHg}$; (d) critically severe, with any of the

\section{What's known}

- Hypoalbuminemia has been frequently observed in patients with SARS-CoV-2 infection.

- Its relationship with the clinical outcome still needs to be clarified.

\section{What's new}

- We provide evidence on a strong association between hypoalbuminemia inflammation markers and clinical outcome. Proteinuria is underreported in SARS-CoV-2 infection; we report it to be a common finding in patients with COVID-19, possibly sustained by systemic and renal endothelial leakage.

- Protein loss might represent a possible biomarker of local and systemic inflammation worthwhile to evaluate disease severity in COVID-19.

following: respiratory failure needing mechanical ventilation, shock, or a combination of other organ failures requiring intensive care. ${ }^{6}$

TABLE 1 Characteristics of the study population

\begin{tabular}{|c|c|c|c|c|}
\hline Characteristic & Total $(n=207)$ & Albumin $<30 \mathrm{~g} / \mathrm{L}(\mathrm{n}=105)$ & Albumin $\geq 30 \mathrm{~g} / \mathrm{L}(\mathrm{n}=102)$ & $P$ value \\
\hline Age, median y (IQR) & $60.90(49.17-71.29)$ & $68.13(59.43-75.16)$ & $55.59(44.55-64.10)$ & $<.001$ \\
\hline \multicolumn{5}{|l|}{ Comorbidities } \\
\hline Hypertension, n (\%) & $71(34.3)$ & $46(43.8)$ & $25(24.5)$ & .003 \\
\hline Diabetes, n (\%) & $28(13.5)$ & $18(17.1)$ & $10(9.8)$ & .155 \\
\hline Mild & $30(14.5)$ & $5(4.8)$ & $25(24.5)$ & \multirow[t]{4}{*}{$<.001$} \\
\hline Moderate & $100(48.3)$ & 39 (37.1) & $61(59.8)$ & \\
\hline Severe & $72(34.8)$ & $56(53.3)$ & $16(15.7)$ & \\
\hline Critically severe & $5(2.4)$ & $5(4.8)$ & $0(0.0)$ & \\
\hline Venturi-type mask & $46(22.2)$ & $38(36.2)$ & $8(7.8)$ & \multirow{3}{*}{$<.001$} \\
\hline C-PAP & $26(12.6)$ & $18(17.1)$ & $8(7.8)$ & \\
\hline Invasive ventilation & $5(2.4)$ & $5(4.8)$ & $0(0.0)$ & \\
\hline Creatinine, mg/dL, median (IQR) & $0.93(0.77-1.10)$ & $0.98(0.77-1.14)$ & $0.93(0.78-1.08)$ & .433 \\
\hline eGFR $_{\text {MDRD }}{ }^{a}$, median (IQR) & $83.01(69.00-96.74)$ & $78.62(65.66-96.46)$ & 85.89 (73.61-96.78) & .076 \\
\hline C-reactive protein, mg/L, median (IQR) & $47.60(19.00-113.00)$ & $94.70(41.00-161.60)$ & $27.30(10.08-54.35)$ & $<.001$ \\
\hline D-dimer, $\mu \mathrm{g} / \mathrm{L}$, median (IQR) & $935.50(528.50-1972.25)$ & $1229.00(919.25-3017.00)$ & $617.00(385.25-1054.25)$ & $<.001$ \\
\hline Interlukin-6, pg/mL, median (IQR) & $69.00(42.50-142.75)$ & $75.50(48.25-140.25)$ & $57.50(38.75-148.00)$ & .437 \\
\hline
\end{tabular}

Abbreviation: C-PAP, continuous positive airway pressure.

Statistically significant $P$ values are reported in bold.

${ }^{a} \operatorname{eGFR}_{\text {MDRD }}\left(\mathrm{mL} / \mathrm{min} / 1.73 \mathrm{~m}^{2}\right)$. 
FIGURE 1 Serum albumin levels at hospital admission in patients with COVID-19 pneumonia according with disease severity $(A)$ and according with clinical outcome (B)
(A)

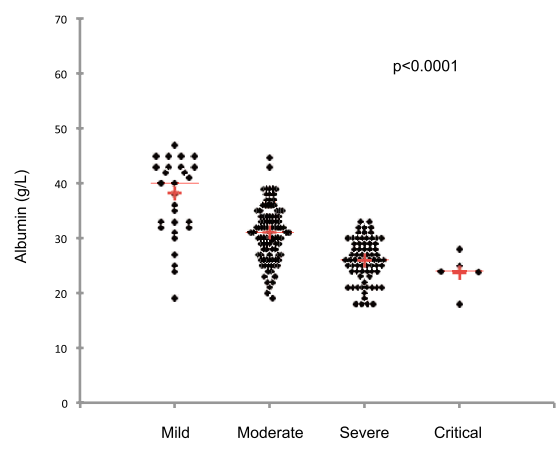

(B)

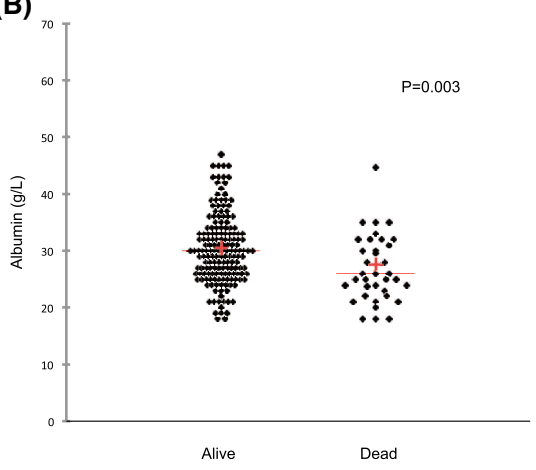

\section{1 | Statistical analysis}

Continuous variables were expressed as medians and interquartile range (IQR) and were compared using nonparametric tests (Wilcoxon's rank-sum or Kruskal-Wallis test). For the categorical variables, the $\chi^{2}$ test was used, or Fisher's exact test when needed.

Non-parametric analysis of the correlation between serum albumin levels and C-reactive protein, d-dimer, interleukin-6 and eGFR was assessed by Spearman's rank correlation coefficient $r_{s}$.

\section{3 | RESULTS}

Two hundred and twenty-two consecutive patients with confirmed COVID-19 with a 30 days follow-up were evaluated. Fifteen subjects were excluded from further analyses because of pre-existing kidney disease. The characteristics of the 207 patients enrolled are reported in Table 1. Serum albumin levels $<30 \mathrm{~g} / \mathrm{L}$ were found in 105 (50.7\%) patients at hospital admission. Overall, the median albumin value was $29.5 \mathrm{~g} / \mathrm{L}$ (IQR 25-32.8): $33 \mathrm{~g} / \mathrm{L}$ (31-37) in patients with albumin $\geq 30$ and $25 \mathrm{~g} / \mathrm{L}(23-27)$ in those with albu$\min <30 \mathrm{~g} / \mathrm{L}$. By the comparison of patients with serum albumin $\geq 30 \mathrm{~g} / \mathrm{L}$ or $<30 \mathrm{~g} / \mathrm{L}$, the latter were older, more frequently affected by arterial hypertension and showed a more serious feature of COVID-19, with higher need for oxygen support and higher inflammatory markers.

A negative association was found between albumin levels and severity of COVID-19 disease $(P<.0001)$ and death $(P=.003)$, see Figure 1.

A significant inverse correlation was observed between albumin and both CRP and D-dimer values at hospital admission by the Spearman coefficient correlation $\left(r_{\mathrm{s}}=-.487\right.$ and $r_{\mathrm{s}}=-.479$, respectively; $P<.0001$ ), while the correlation between albumin levels and IL-6 showed a non-significant trend $\left(r_{s}=-.215 ; P=.066\right)$ (Figure 2).

Finally, a positive correlation was found between albumin levels and eGFR ( $\left.r_{s}=.184 ; P=.008\right)$ (Figure 3 ).

Over a 7-days observation from hospitalisation, albumin levels were stable in patients with an improving clinical course over the
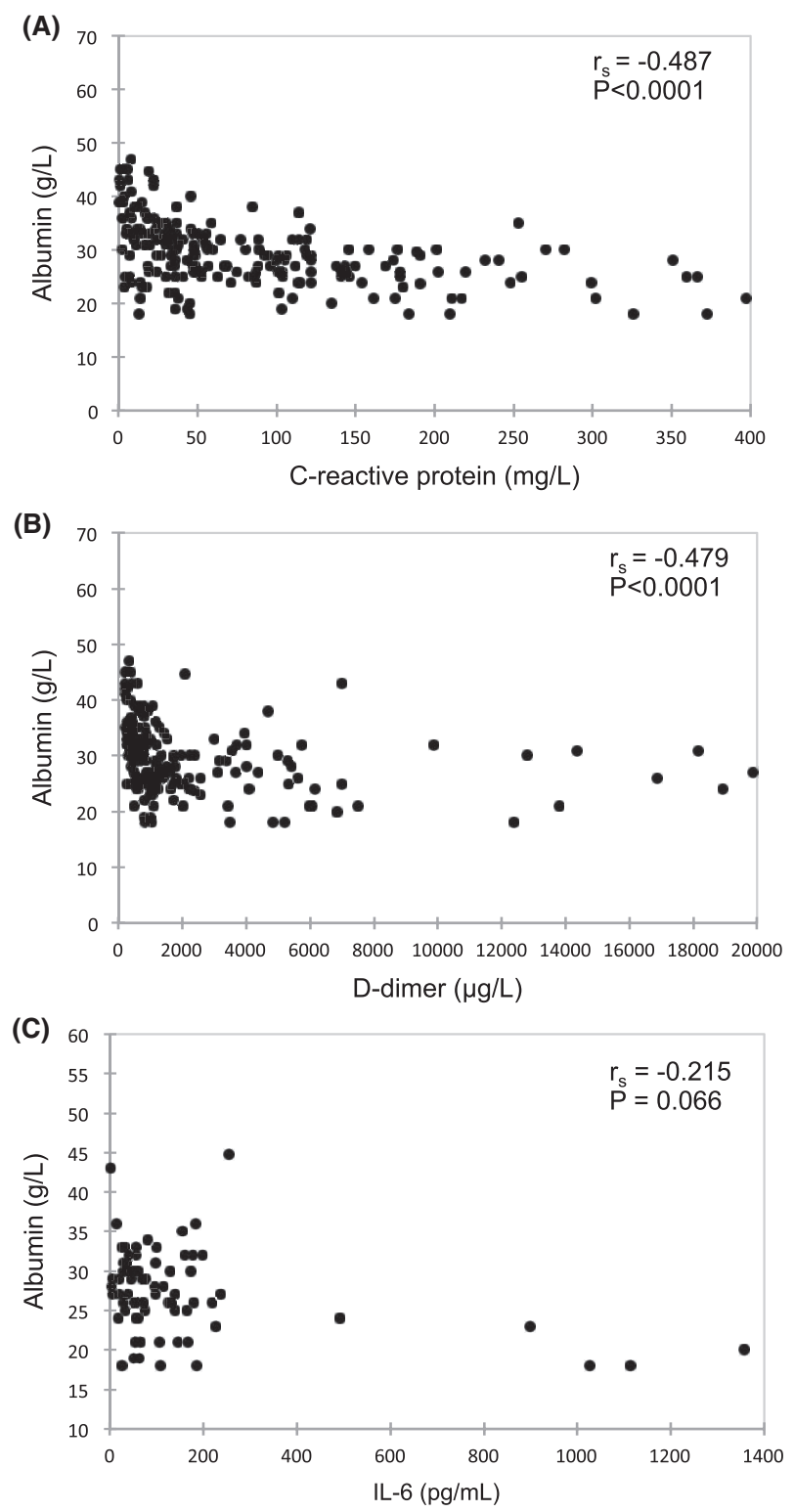

FIGURE 2 Spearman coefficient correlation between serum albumin at admission and C-reactive protein (A) levels, D-dimer levels (B) and serum IL-6 levels (C) at hospital admission in patients with COVID-19 pneumonia 
follow-up, while a persistent decrease occurred in patients eventually undergoing mechanical ventilation or death (medians of delta albumin $0 \mathrm{~g} / \mathrm{L}$ and $-9 \mathrm{~g} / \mathrm{L}$, respectively; $P=.001$ ) (Figure 4).

A complete urine examination was available for 136 of 207 patients. Proteinuria was observed in 102 (75\%) patients, with a protein loss in the order of $50 \mathrm{mg} / \mathrm{dL}$ in $63 \%$ and of $100 \mathrm{mg} / \mathrm{dL}$ in $12 \%$. Proteinuria did not significantly differ between patients with hypoalbuminemia and those with albumin $\geq 30 \mathrm{~g} / \mathrm{L}$ (81\% and $67 \%$, respectively; $P=.09$ ).

Twenty-five patients (18\%) presented mild hematuria (between $0.5-1 \mathrm{mg} / \mathrm{dL}$ ), while leukocyturia was present in 23 patients (17\%) at the following extent: 25 leukocytes/ $\mu \mathrm{L}$ in 11 (8\%), 50-75 leukocytes/ $\mu \mathrm{L}$ in 5 (3.6\%), 250 leukocytes/ $\mu \mathrm{L}$ in 4 (3\%) and 500 leukocytes/ $\mu \mathrm{L}$ in $3(2.2 \%)$.

\section{4 | DISCUSSION}

Several papers have described hypoalbuminemia in patients with COVID-19. ${ }^{1-4}$ However, the underlying mechanisms have not been clarified, yet. Here, we report the prevalence of hypoalbuminemia in a cohort of 207 patients with COVID-19 and its relationship with disease severity and clinical outcome. As previously demonstrated, ${ }^{7,8}$

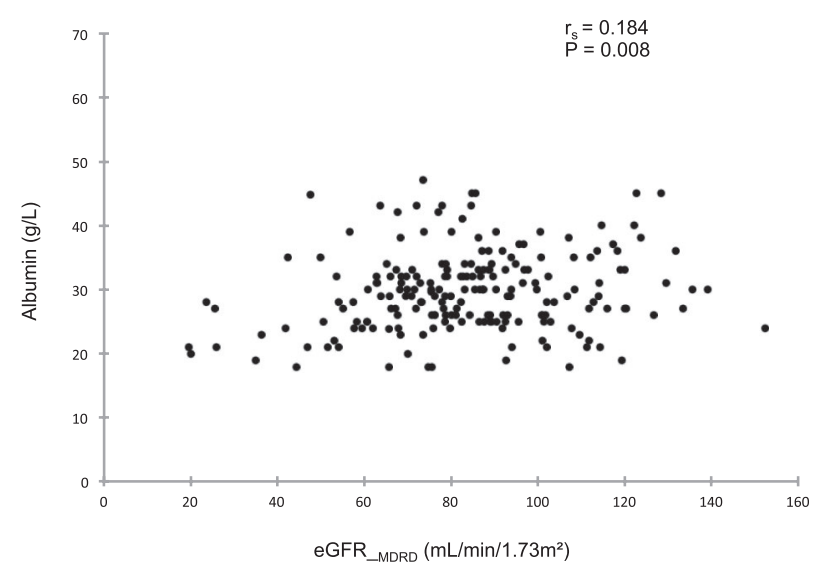

FIGURE 3 Spearman coefficient correlation between serum albumin and eGFR (MDRD) at hospital admission in patients with COVID-19 pneumonia the disease severity and death strongly correlate with low levels of serum albumin at admission. Furthermore, we observed a negative association between albumin levels and inflammation markers (CRP and D-dimer).

Several pathogenic mechanisms may be implicated in the decreased serum albumin of patients with COVID-19. (a) transcapillary leakage: under inflammatory conditions the physiological trancapillary escape rate of albumin may increase of several fold as observed in acute respiratory distress syndrome (ARDS) and sepsis, possibly mediated by interleukin-2, interferon- $\alpha$ and interleukin-6. This disruption of endothelial integrity leads to albumin leakage into the pulmonary interstitium where the protein concentration increases from less than $40 \%$ to more than $60 \%$ of the plasma value. Consequently, serum albumin concentration decreases in many critically ill patients. ${ }^{9-11}$ (b) Besides pulmonary sequester, sepsis is also associated with the disruption of the glomerular filtration barrier which leads to increased permeability to albumin and its leakage into urine. ${ }^{12}$ (c) Histopathological examination of kidney from autopsy samples demonstrated tubular necrosis, luminal loss of brush border and vacuole degeneration which may be partially caused by the direct cytopathic effect of SARS-CoV-2, which particles were found in proximal tubular epithelium. $^{13}$

COVID-19 severe infection is characterised by a high cytokine release with a hyperinflammatory state that can induce endothelial damage even before the onset of a definite ARDS. ${ }^{1,14}$ In our cohort of patients with COVID-19, low levels of serum albumin were very common and were observed in quite an early stage of pulmonary disease. Indeed, $41.9 \%$ of hypoalbuminemic patients at hospital admission were on none or mild oxygen support (nasal cannula) and did not fulfill the diagnostic criteria of ARDS. ${ }^{15}$ In a recent remarkable paper, high renal tropism of SARS-CoV-2 was demonstrated and it was suggested as a possible explanation of kidney injury even in patients with mild COVID-19 infection. ${ }^{16}$ However, we confirmed here a strong association of hypoalbuminemia with respiratory impairment and disease severity as well as with inflammatory state.

As far as urinalysis is concerned, a mild to moderate proteinuria was frequently observed in our cohort, independently from the level or serum albumin levels, thus reinforcing the hypothesis of a
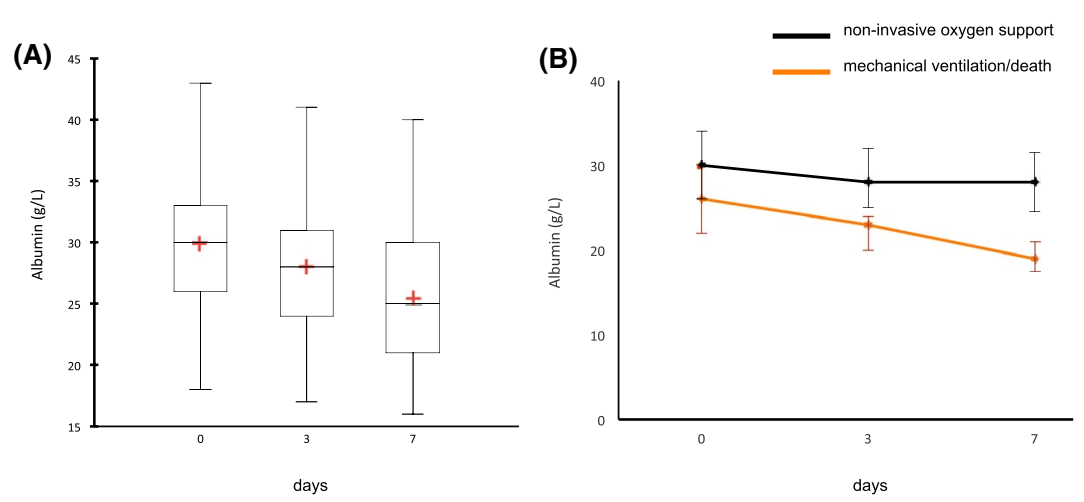

FIGURE 4 Median (IQR) serum albumin values at admission, day 3 and day 7 of hospitalisation in the whole cohort (A) and stratified by clinical outcome (B). Medians of delta albumin: $P=.001$ 
systemic endothelial leakage rather than a prevailing renal tubular damage. This is also supported by the observation of a low rate and mild extent of hematuria and leukocyturia.

The main limitation of the study is represented by the lack of urinalysis in a number of patients and the unavailability of a 24-hour urine collection that could have allowed a more accurate definition of proteinuria. In addition, we could not evaluate an appropriate trend of serum albumin on a long follow-up period because of the retrospective nature of our study that did not allow a systematic approach to the timing of laboratory markers.

In summary, hypoalbuminemia is common amongst COVID-19 patients and is strictly associated with inflammation markers and clinical outcome. The common finding of proteinuria, even in the absence of creatinine increase, indicates protein loss as a possible biomarker of local and systemic inflammation worthwhile to evaluate disease severity in COVID-19.

\section{DISCLOSURE}

The authors have declared no conflicts of interest for this article.

\section{ORCID}

Laura Milazzo iD https://orcid.org/0000-0003-3039-3039

\section{REFERENCES}

1. Chen G, Wu D, Guo W, et al. Clinical and immunological features of severe and moderate coronavirus disease 2019. J Clin Invest. 2020;130:2620-2629.

2. Huang C, Wang Y, Li X, et al. Clinical features of patients infected with 2019 novel coronavirus in Wuhan, China. Lancet. 2020;8:475-481.

3. Chen N, Zhou M, Dong X, et al. Epidemiological and clinical characteristics of 99 cases of 2019 novel coronavirus pneumonia in Wuhan, China: a descriptive study. Lancet. 2020;395:507-513.

4. Henry BM, de Oliveira MHS, Benoit S, Plebani M, Lippi G. Hematologic, biochemical and immune biomarker abnormalities associated with severe illness and mortality in coronavirus disease 2019 (COVID-19): a meta-analysis. Clin Chem Lab Med. 2020;58:1021-1028.
5. Levey AS, Coresh J, Balk E, et al. National Kidney Foundation practice guidelines for chronic kidney disease: evaluation, classification, and stratification. Ann Intern Med. 2003;139:137-147.

6. Xu YH, Dong JH, An WM, et al. Clinical and computed tomographic imaging features of novel coronavirus pneumonia caused by SARSCoV-2. J Infect. 2020;80:394-400.

7. Gong J, Ou J, Qiu X, et al. A tool to early predict severe corona virus disease 2019 (COVID-19): a multicenter study using the risk nomogram in Wuhan and Guangdong, China. Clin Infect Dis. 2020;71:833-840.

8. Cheng Y, Luo R, Wang K, et al. Kidney disease is associated with in-hospital death of patients with COVID-19. Kidney Int. 2020;97:829-838.

9. Sartori C, Rimoldi SF, Scherrer U. Lung fluid movements in hypoxia. Prog Cardiovasc Dis. 2010;52:493-499.

10. Ware LB, Fremont RD, Bastarache JA, Calfee CS, Matthay MA. Determining the aetiology of pulmonary oedema by the oedema fluid-to-plasma protein ratio. Eur Respir J. 2010;35:331-337.

11. Gonzales JN, Lucas R, Verin AD. The acute respiratory distress syndrome: mechanisms and perspective therapeutic approaches. Austin J Vasc Med. 2015;2:1009. PMID:26973981.

12. Bomsztyk K, Mar D, An D, et al. Experimental acute lung injury induces multi-organ epigenetic modifications in key angiogenic genes implicated in sepsis-associated endothelial dysfunction. Crit Care. 2015;19:225.

13. Su H, Yang M, Wan C, et al. Renal histopathological analysis of 26 postmortem findings of patients with COVID-19 in China. Kidney Int. 2020;98:219-227.

14. Ruan Q, Yang K, Wang W, Jiang L, Song J. Clinical predictors of mortality due to COVID-19 based on an analysis of data of 150 patients from Wuhan, China. Intensive Care Med. 2020;46:846-848.

15. Definition Task Force ARDS, Ranieri VM, Rubenfeld GD, et al. Acute respiratory distress syndrome: the Berlin Definition. JAMA. 2012;307:2526-2533.

16. Puelles VG, Lütgehetmann M, Lindenmeyer MT, et al. Multiorgan and renal tropism of SARS-CoV-2. N Engl J Med. 2020;383:590-592.

How to cite this article: Bassoli C, Oreni L, Ballone E, et al. Role of serum albumin and proteinuria in patients with SARS-CoV-2 pneumonia. Int J Clin Pract. 2020;00:e13946. https://doi.org/10.1111/ijcp.13946 